\title{
Travaux sur Pascal et le vide au Japon
}

\section{Hiroki Takeda}

\section{OpenEdition}

Journals

Édition électronique

URL : http://journals.openedition.org/ccibp/500

DOI : $10.4000 /$ ccibp. 500

ISSN : 2493-7460

\section{Éditeur}

Centre international Blaise Pascal

\section{Édition imprimée}

Date de publication : 15 décembre 2007

Pagination : $40-48$

ISBN : 9782845163720

ISSN : 0249-6674

\section{Référence électronique}

Hiroki Takeda, "Travaux sur Pascal et le vide au Japon », Courrier du Centre international Blaise Pascal [En ligne], 29 | 2007, mis en ligne le 02 décembre 2015, consulté le 15 septembre 2020. URL : http:// journals.openedition.org/ccibp/500

Ce document a été généré automatiquement le 15 septembre 2020

Centre international Blaise Pascal 


\title{
Travaux sur Pascal et le vide au Japon
}

\author{
Hiroki Takeda
}

\section{NOTE DE L'ÉDITEUR}

Le Japon est depuis plusieurs années le lieu d'une recherche continue, principalement animée par Kimiyo Koyanagi, sur les expériences de Pascal sur le vide. Le jeudi 4 mai 2006, Mme Koyanagi est venue avec H. Takeda à la Maison des Sciences de l'Homme de Clermont-Ferrand, présenter publiquement le DVD que nos amis japonais ont réalisé sur la reconstitution des diverses formes de l'expérience du vide dans le vide. Cette réalisation, permet de suivre pas à pas la procédure nécessaire pour effectuer les expériences de Pascal. Il faut espérer qu'elle fera l'objet d'une publication prochaine, car elle constitue un document très instructif qui pourrait être mis à profit non seulement par les chercheurs et les amis de Pascal, mais dans le cadre pédagogique de l'enseignement secondaire. Nous publions ici d'une part le compte-rendu condensé de ces travaux, rédigé par $\mathrm{H}$. Takeda, ainsi qu'une étude du même auteur sur la manière dont Pascal aborde la question de l'élasticité de l'air.

\section{NOTE DE L'AUTEUR}

Expériences effectuées par Kimiyo KOYANAGI, Hidetaro HONDA, Hiroki TAKEDA, Masao UCHIDA, Haruo NAGASE, Eio HONMA. 


\section{Compte rendu de la reconstitution des expériences du vide dans le vide}

1 Nous avons reconstitué, en été 2004, les « expériences du vide dans le vide » effectuées par Pascal et ses contemporains. Nous remercions profondément, d'abord le Ministère de l'Éducation et de la Recherche japonais qui a subventionné nos études, ensuite le Tôyô-ji, temple bouddhiste de l'école Ippen-Shû, qui a eu la gentillesse de nous offrir généreusement le lieu de l'expérience et de soutenir sans trêve nos activités et enfin tous ceux qui ont assisté aux expériences et qui ont contribué à la réalisation par leurs précieux commentaires.

2 Ces quatre expériences du vide dans le vide, hormis le deuxième dispositif de Pascal, avaient déjà été reconstituées en 1982 par Kimiyo Koyanagi et Masao Uchida d'après les descriptions originales au laboratoire de transformation du verre de M. Shigeru Andô à Tokyo. Nous avons utilisé, cette fois-ci, les mêmes dispositifs fabriqués à cette époque. Sur leurs plans et la manipulation détaillée des expériences, voir le Courrier du CIBP, 11, 1989, p. 2-23, ou «The feasibility of les Expérience du vide dans le vide as contrived by Pascal and his contemporaries" in Réflexions sur la place de Descartes et de Pascal dans l'histoire de la pensée scientifique et Étude des bibliographies concernées, p. 81-106. Alors que la reconstitution de 1982, réalisée par Koyanagi, Uchida et M. Kôji Tsunoda, assistant d'expérience, n'avait pas été publique, nous avons effectué cette fois-ci la reconstitution devant d'autres participants intéressés et enregistré sur DVD la quasitotalité des processus de l'expérience.

Seul l'emploi de la vessie de porc pour boucher les extrémités des tuyaux s'est avéré trop difficile à cause de la grande pression exercée par le mercure. Il nous a fallu remplacer les vessies par des bouchons de caoutchouc utilisés pour la chromatographie des gaz. Sur l'usage des vessies à cette époque-là, voir KIMIYo Koyanagi, « Recherche sur les «vessies» et "ballons» en tant qu'instruments d'expérience. Démarche et tâtonnement » rédigé par KIMIYO Koyanagi avec la collaboration de MASAO Uchida, in Réflexions sur la place de Descartes et de Pascal dans l'histoire de la pensée scientifique et Etude des bibliographies concernées, p. 107-143.

\section{L'expérience de Pascal, premier dispositif de Pascal}

Pascal fait allusion à cette expérience dans sa lettre à Périer du 15 novembre 1647 ( Euvres complètes de Pascal, éd. Jean Mesnard, Desclée de Brouwer, "Bibliothèque européenne », t. II, 1970, p. 679). Le P. Noël donne dans la Gravitas comparata une description plus précise (voir Cuvres complètes, II, pp. 635-636).

Données expérimentales :

Altitude : $163 \mathrm{~m}$. Le 23 août 2004, $14 \mathrm{~h}$

Pression : $1013.1 \mathrm{mb}$

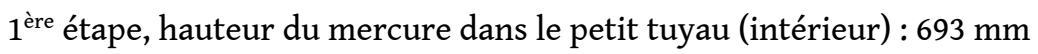

$2^{\text {ème }}$ étape, hauteur du mercure dans le grand tuyau (extérieur) : $730 \mathrm{~mm}$ 
Hauteur du mercure dans le petit tuyau (intérieur) : $693 \mathrm{~mm}$

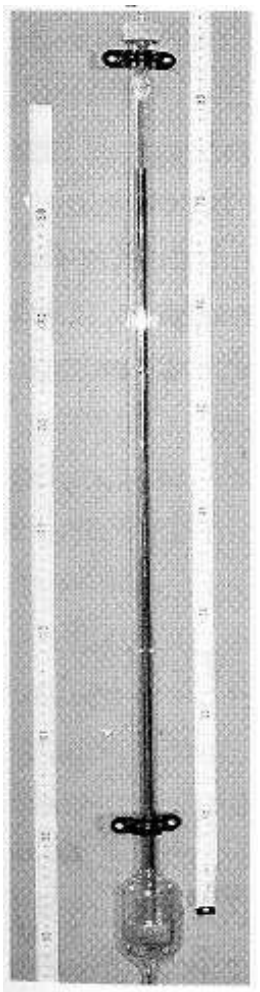

6 Le point le plus énigmatique de cette expérience réside dans le fait qu'il n'est pas nécessaire de fermer l'orifice inférieur du petit tuyau pour pratiquer l'expérience de Torricelli dans l'espace vide. Pourquoi Pascal a-t-il voulu le fermer malgré les grandes difficultés de la manipulation? Sans doute, pensons-nous, pour cette raison que l'intérêt de Pascal et de ses contemporains s'est concentré sur la nature de l'espace vide, c'est-à-dire sur le phénomène se produisant dans le vide «apparent ». Afin de satisfaire cet intérêt, il a fallu que Pascal procède à l'expérience de Torricelli dans le grand tube une fois vide, autrement dit, qu'il ait commencé par l'état où se trouve, dans le grand tube vide, le petit tuyau rempli de vif-argent. Si on ne bouche pas l'extrémité inférieure du petit tuyau, le vif-argent présent dans le petit tuyau descend, en même temps que tombe le vif-argent remplissant le grand tube (n'oublions pas que le petit tuyau n'est pas visible à cause du mercure présent dans le grand tube), de sorte que les spectateurs ne peuvent observer, au sens rigoureux du terme, l'expérience de Torricelli dans le vide.

\section{L'expérience de Roberval}

7 L'expérience de Roberval nous est connue par une brève mention de l'auteur dans la De vacuo narratio et par la Gravitas comparata du P. Noël; le texte est cité d'après l'édition des Euvres complètes, II, pp. 637-639.

8 Le dispositif de Roberval abandonne déjà l'idée du premier modèle de Pascal qui s'en tenait à l'expérience du vide " dans le vide », le dispensant ainsi des manipulations très complexes que Pascal avait effectuées dans sa première expérience. Il n'est pas nécessaire d'insérer le petit tuyau dans le tube vide ou de laisser vide le tube dans lequel on insère le petit tuyau rempli de vif-argent. 
Le petit tuyau pénètre dans un flacon de verre en forme de cœur humain. Suivant la pression de l'air exercée à l'intérieur du flacon, on pourra bien observer que le vifargent présent dans le tuyau monte ou descend. Il est probable que, grâce à l'amélioration apportée par Roberval, la réalisation de l'expérience est devenue plus facile.

Ce dispositif présente toutefois deux difficultés. Premièrement, il est très difficile de remplir de vif-argent la partie supérieure du flacon puisque celui-ci est en forme de cœur humain, selon le texte du P. Noël. Nous avons dû effectivement incliner le dispositif pour enlever l'air restant dans la partie supérieure du flacon et l'aspirer à l'aide d'une pipette. De l'air demeurant dans le flacon et se transformant en bulles lorsqu'on exerce l'expérience, monte peu à peu dans le tuyau rempli de vif-argent. Ce phénomène aurait plaidé fort probablement en faveur des "plénistes" qui pensaient qu'il n'y a pas d'espace vide dans ce monde.

Deuxièmement, les trois joints utilisés affaiblissent beaucoup la structure du dispositif. Le vif-argent, 13,6 fois plus pesant que l'eau, tend à sortir sans cesse de la partie plus faible du dispositif, à cause de quoi notre reconstitution s'est terminée par un échec à l'étape où il s'agissait de verser le vif-argent. Rappelons que le deuxième dispositif de Pascal n'a pas de joints; Pascal s'en vante. Pareil échec aurait bien pu se passer en fait au XVII ${ }^{e}$ siècle. Nous pensons en tout cas que le succès dans la réalisation de cette expérience était, en fait, plus difficile qu'on ne l'imaginait.

Le petit tuyau pénètre dans un flacon de verre en forme de cœur humain.

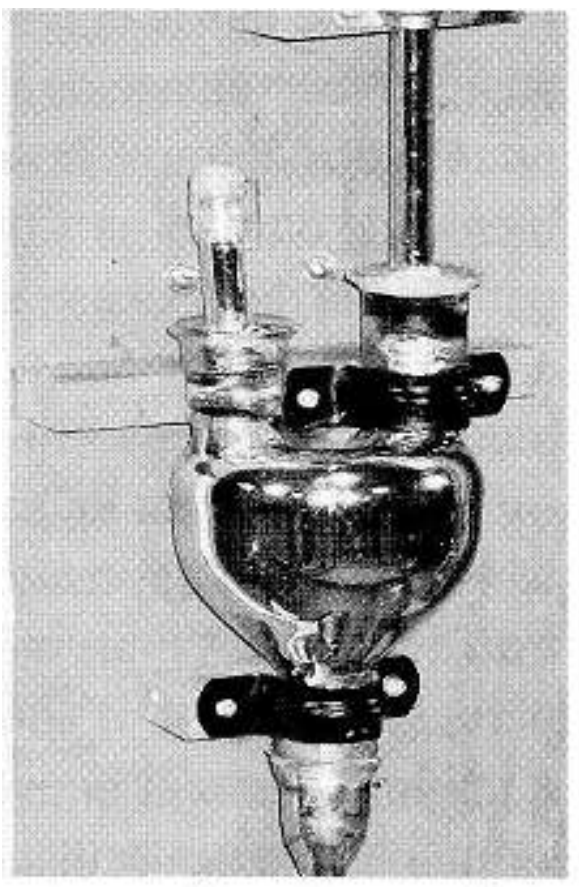




\section{L'expérience de Pascal d'après Le Traité de la pesanteur de la masse de l'air (chapitre VI)}

12 Ce dispositif a été restitué en 1974 par feu Monsieur Akira Kurobe au Laboratoire National de Métrologie de Tokyo. Pour faciliter l'expérience, il a remplacé la vessie de porc, par trois robinets équipant le tuyau.

L'édition originale du traité de Pascal présente une figure défectueuse (voir CEuvres complètes, op.cit., II, p. 1087, et le commentaire, p. 1042).

Données expérimentales :

Altitude : $163 \mathrm{~m}$

Le 24 août $2004,12 \mathrm{~h}$

Pression : $1006.2 \mathrm{mb}$

$1^{\text {ère }}$ étape : $744 \mathrm{~mm}, 2^{\text {ème }}$ étape $729 \mathrm{~mm}$

15 L'idée de ce dispositif est évidemment empruntée à Roberval. Pour qu'il soit original, il aurait suffi à Pascal d'inverser la droite et la gauche dans la figure du dispositif et de changer la forme du flacon. Pascal lui-même, malgré cela, ne mentionne pas le nom de Roberval et se comporte comme s'il s'était inspiré directement de Torricelli. Cette attitude qui ne fait pas référence aux précédents travaux auxquels on est redevable, semble assez commune chez les savants du XVII ${ }^{e}$ siècle, non seulement chez Pascal, mais aussi chez Roberval et Auzout.

16 Le mérite du deuxième dispositif de Pascal consiste à inventer un dispositif totalement solidaire en supprimant les joints qui pouvaient provoquer de grandes difficultés comme dans le modèle de Roberval. Pascal insiste en effet sur ce point dans son texte, en proclamant que « les deux tuyaux (...) n'en font proprement qu'un, puisqu'ils ont communication l'un dans l'autre ».

17 Quant à la deuxième étape, le texte témoigne du fait que «le doigt qui bouche son ouverture l'en garde, il arrive en récompense que le doigt souffre beaucoup de douleur ». En dépit du témoignage de Pascal, plutôt que de "la douleur », nous avons éprouvé la sensation que le doigt était comme bien collé ou fixé sur l'orifice de tuyau. Certains pensent à partir de là que l'expression exagérée "la douleur " visant à souligner la force de la pression de la masse de l'air était plutôt le fait de Pascal l'homme de lettres.

Sur la question de savoir si l'expérience du vide dans le vide d'après Le traité de la pesanteur de la masse de l'air (chapitre VI) a vraiment été effectuée ou non, les six participants sont d'accord, malgré certaines nuances. Kimiyo Koyanagi pense que, malgré son élégance et la simplicité de son principe, ce dispositif correspond à une expérience de pensée (voir K. Koyanagi, «Pascal et l'expérience du vide dans le vide »). Masao Uchida était autrefois plus prudent sur ce type de question. Son article paru en 1982, « De la faisabilité des Expériences du vide dans le vide rapportées par Pascal et ses contemporains ", critiquait du point de vue de la faisabilité, l'attitude qui présumait, sans procéder à un examen scrupuleux, qu'il s'agissait d'une expérience de pensée. De nos jours, en se fondant sur les études achevées par Kimiyo Koyanagi sur le plan à la fois des expériences reconstituées et des analyses des textes, il affirme avec assurance que la description du Traité de la pesanteur de la masse de l'air ne peut jamais constituer un rapport correspondant à une expérience effectivement réalisée et pense que l'esprit génial de Pascal se rend compte du principe de l'équilibre des liqueurs jusqu'au point le 
plus délicat et le développe précisément dans son écriture de façon inductive. Deux chercheurs, Hidetaro Honda et Haruo Nagase, approuvent, pour le moment, l'idée émise par Koyanagi et Uchida. Hiroki Takeda, admet la très forte probabilité d'une expérience de pensée, sans nier la possibilité que cette expérience ait été réellement effectuée. On ne saurait jamais répondre de façon décisive à cette question à moins de découvrir le dispositif effectivement employé par Pascal ou les documents de sa fabrication.

"Les deux tuyaux (...) n'en font proprement qu'un, puisqu'ils ont communication l'un dans l'autre ».

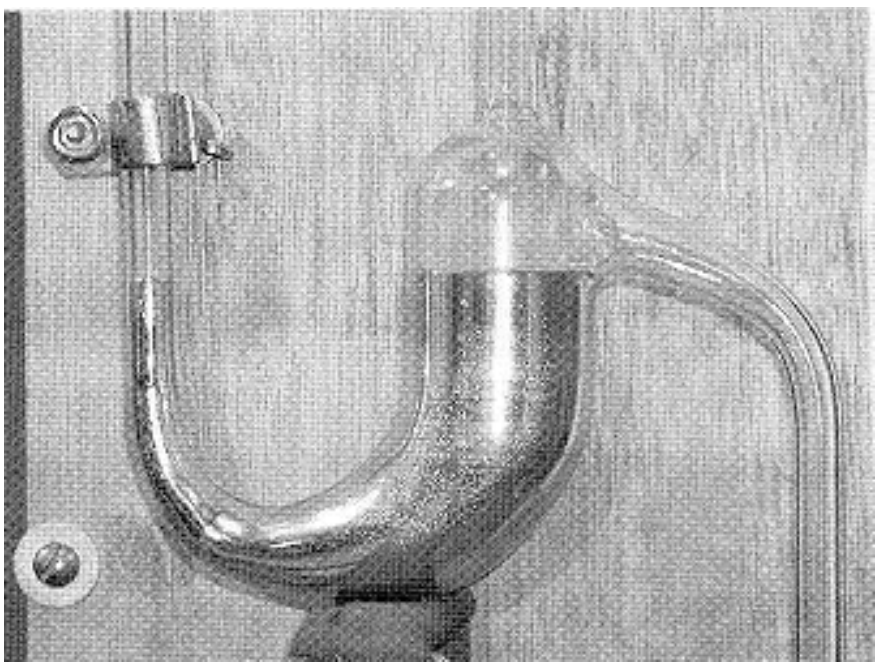

De toute façon, les participants sont unanimes à penser qu'il n'est pas possible d'aboutir à une conclusion définitive en ayant seulement recours à la reconstitution des expériences, et qu'il faut arriver à la plus haute vraisemblance possible, en prenant en considération toutes les informations disponibles : l'existence de témoins, la situation de l'époque, la personnalité de Pascal, etc. Réciproquement, la reconstitution des expériences historiques donne une occasion de réfléchir, de façon globale, sur les diverses données se rapportant à l'exécution des expérimentations. Ce qui est, croientils, la signification principale à accorder à la reconstitution d'une expérience.

\section{L'expérience de Rohault}

\section{Commentaire :}

Voir Rohault, Traité de Physique (1671), pp. 92-95.

21 Données expérimentales :

Altitude : $163 \mathrm{~m}$

Le 24 août 2004, $10 \mathrm{~h}$

Pression : $1006.8 \mathrm{mb}$

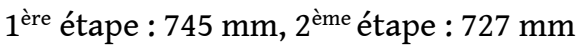

22 Le dispositif de Rohault, qui provient à coup sûr de celui de Pascal, permet la manipulation encore plus facilement. Nous en avons confirmé la facilité dans notre reconstitution et avons en fait presque obtenu les valeurs théoriques. Ce dispositif a certainement été présenté, à plusieurs reprises, dans le «Mercredi » qu'organisait Rohault. 


\section{L'expérience de Rohault}

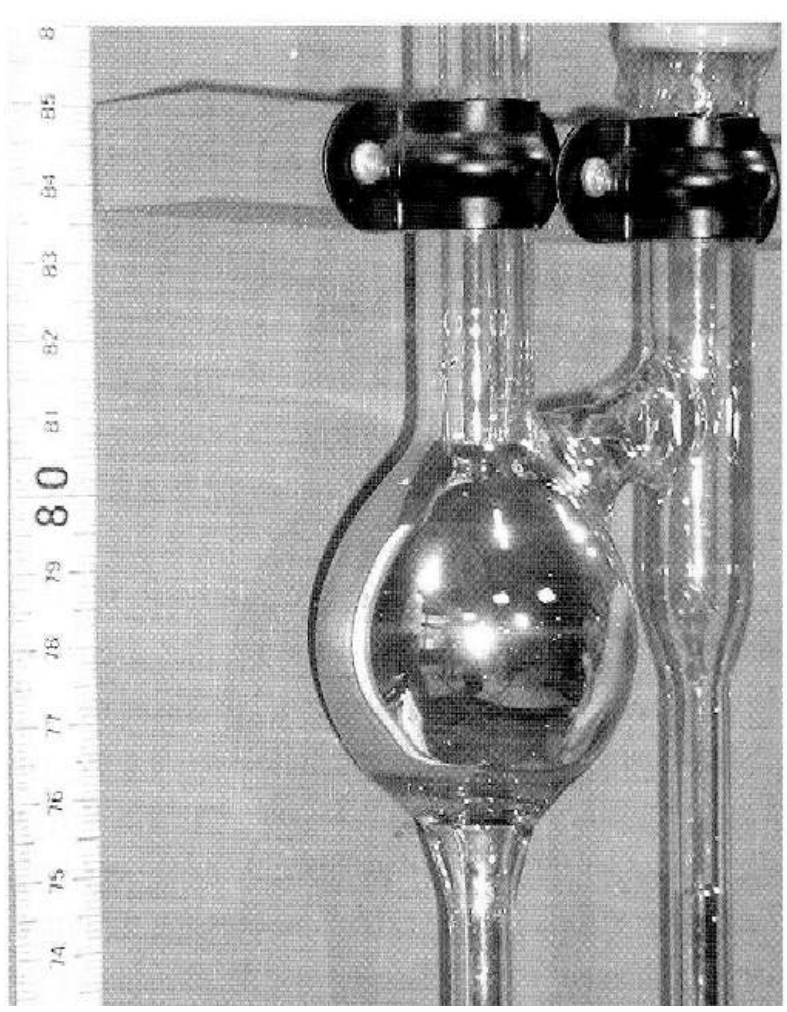

\section{Pascal, Roberval et l'élasticité de l'air}

Pascal et Roberval, deux savants également intrigués par l'expérience de Torricelli, ont eu des opinions divergentes sur la théorisation de l'expérience. Tandis que Pascal s'est concentré sur l'établissement d'une théorie cohérente, s'appuyant, comme l'indique le titre de son ouvrage, sur les principes de «l'équilibre des liqueurs » et de « la pesanteur de la masse de l'air ", Roberval, introduisant la notion de " elater ", à savoir d'élasticité de l'air, s'est efforcé de mettre en lumière le rapport entre cette dernière et la pression d'air. Notre article, portant sur la notion d'élasticité de l'air, met en avant leurs manières d'apprivoiser cette notion et de la situer de façon cohérente dans leur système physique, et montre comment se construit la philosophie naturelle chez ces deux savants.

La première section sera consacrée à l'origine du problème de l'élasticité de l'air, qui se trouve déjà dans les commentaires de Torricelli sur l'expérience qu'il a effectuée luimême. La seconde sera réservée à la découverte de l'élasticité de l'air par Roberval et à la modélisation de Jean Pecquet, qui nous semble utile pour mieux aborder la structure du Traité de la pesanteur de la masse de l'air. Après avoir ainsi examiné le développement de l'idée de l'élasticité, nous envisagerons dans la troisième partie l'attitude de Pascal face à l'élasticité de l'air. 


\section{L'origine du problème de l'élasticité de l'air} tard, à la compréhension exacte de la pression atmosphérique, à savoir que l'air comprimé au fond de l'atmosphère veut se dilater. Trois notions primordiales pour saisir de manière adéquate le mécanisme de la pression atmosphérique ont été effectivement présentées, sous des formes figurées, dans la réflexion faite par Torricelli en 1644. À savoir la pesanteur de l'air, la pression causée par la pesanteur, et l'élasticité de l'air.

\section{L'élasticité de l'air et les contemporains de Pascal (Roberval et Pecquet)}

\subsection{Roberval et l'élasticité de l'air}

Lorsqu'il a assisté à la répétition de l'expérience de Torricelli, Roberval a remarqué le phénomène suivant: de petites bulles d'air montaient de la partie inférieure du tube (ce qui a été confirmé aussi dans nos expériences ${ }^{3}$ ). Il ne faut pas oublier que les bulles d'air qui se présentent dans le tube n'étaient pas, à cette époque-là, un phénomène secondaire. Au contraire, c'est ce qui a provoqué une discussion essentielle concernant la raréfaction et la condensation de l'air.

Ce phénomène a incité Roberval à réaliser une expérience bien connue en utilisant une vessie de carpe tout aplatie, mise dans la partie vide du tuyau, après avoir été vidée le mieux possible de tout air. La vessie de carpe désenflée, mise dans l'espace en apparence vide, s'enfle tout d'un coup, ce qui a conduit Roberval à reconnaître que la capacité de dilatation de l'air sans aucune chaleur était plus forte qu'on ne l'avait imaginé. De ces deux phénomènes, d'une part la raréfaction de l'air, d'autre part « de

Courrier du Centre international Blaise Pascal, 29 | 2007 
petites gouttes d'air» qui montent de la partie inférieure du tube et «au fur et à mesure de la montée, grandissent de plus en plus ${ }^{4}$ ", Roberval tire la conclusion que la partie supérieure du tube, qui apparaissait comme vide, est en réalité remplie d'air raréfié. La philosophie naturelle de Roberval admet, il est vrai, l'existence de l'espace vide qui précède la création du monde par Dieu ${ }^{5}$, mais l'expérience de Torricelli ne l'a pas autorisé à conclure de façon indubitable que l'espace vide surgissait dans la réalité. Dès lors, la réflexion sur l'expérience de Torricelli glisse du problème de l'existence du vide vers la théorie de matière, qui, avec la notion d'espace, forme une problématique.

Roberval, n'ayant pas cessé ses recherches sur l'élasticité de l'air, a tenté deux autres expériences comparatives utilisant celle de Torricelli. L'une est celle qui consiste à mettre dans la partie supérieure du tuyau la même quantité d'eau et d'air. L'air introduit a fait baisser, selon Roberval, la hauteur du mercure davantage que l'eau, cela bien que l'air soit moins pesant que l'eau. Cette expérience fait comprendre clairement qu'un fluide élastique comme l'air exerce sa pression d'une façon tout à fait différente de celle d'un fluide non-élastique comme l'eau, et par conséquent que la pesanteur de l'air n'est pas la seule cause à produire l'effet de la pression atmosphérique.

32 L'autre expérience consiste à introduire la même quantité d'air dans la partie supérieure de deux tuyaux dont la longueur est différente. L'air mis dans la partie supérieure du tuyau le plus court pousse la colonne de mercure et fait baisser son niveau davantage que l'air mis dans le tuyau le plus long, puisque la capacité de « ressort » de l'air s'exerce plus fortement dans un espace plus restreint. Par ces deux expériences effectuées en 1648 et de façon plus scientifique que Torricelli avec son idée figurée d'un "amas de laine», Roberval a récolté des éléments suffisants pour comprendre que la pression de l'atmosphère n'était pas autre chose que la force de la dilatation de l'air, comprimé à cause de la pesanteur de l'air qui «se couche sur luimême ${ }^{6}$ ».

Alors que les recherches de Roberval sur l'élasticité de l'air ont ouvert la voie aux travaux de Boyle et Mariotte, les écrits qu'il a laissés là-dessus n'ont malheureusement exercé sur ses contemporains qu'une influence moindre, par rapport à la grande réputation de ses expériences ${ }^{7}$. C'est, à mon avis, parce que la propriété d'élasticité n'a pas été expliquée de manière suffisamment cohérente pour convaincre les mécanistes qui représentaient le courant d'idées le plus répandu à ce moment-là. Quelles difficultés la théorie de Roberval présente-t-elle?

Elle pose premièrement le problème de la philosophie naturelle. Roberval, se servant de termes comme "facultas resiliendi ", "vis ad resiliendum ", " innato appetitu », etc., suppose une propriété immanente à chaque élément, c'est-à-dire une «vis naturae elementaris ", douée d'une faculté à la fois active et occulte. Roberval, s'opposant, depuis une dizaine d'années, à une idée stéréotypée du mécanisme qui, comme chez Descartes, a mené à bannir toutes les qualités immanentes au corps, proposa donc hardiment l'idée d'un couple de forces intérieures à la matière, une "attraction mutuelle» invoquée pour bien expliquer l'agrégat des atomes, et une «facultas resiliendi », alléguée à partir de l'expérience de la vessie de carpe. La présupposition de ces «qualités occultes" aurait eu d'autant plus de difficulté à se propager que cela pêchait contre " la géométrisation du monde » à cette époque de mécanisme cartésien.

35 La problématique de Roberval implique, en second lieu, la position d'une hypothèse ad hoc. Roberval présume l'existence d'une autre force venant s'ajouter à l'attraction mutuelle professée depuis le De mundi systemate (1644), publié sous le nom de Samos 
Aristarcos ${ }^{8}$. C'est la faculté de « elater » des éléments. La supposition d'une hypothèse de travail, qui est, en général, il est vrai, le premier pas pour développer une théorie scientifique, empêcherait cependant l'établissement d'un système cohérent des sciences, si on inventait une nouvelle hypothèse chaque fois qu'un nouveau phénomène naturel est découvert. La prise de position sceptique de Roberval, tout en repoussant l'affirmation définitive à l'égard de l'existence du vide dans le monde d'ici-bas, suivant les témoignages des expériences, ne fonctionne plus en face de l'élasticité de l'air. Le scepticisme robervalien ne se caractérise pas donc, comme l'écrit Fanton d'Andon, par " un doute épistémologique », mais par " un doute idéologique ${ }^{9}$ " dans le sens où l'on ne s'arrête pas à un dogme particulier. Roberval soumettra la même idée d'attraction mutuelle à titre d'hypothèse dans le débat sur la cause de la pesanteur tenu à l'Académie royale des Sciences en $1669^{10}$.

Troisièmement, une insistance excessive sur l'élasticité de l'air et l'attraction mutuelle amène Roberval à faire abstraction de l'importance de la pesanteur de l'air. La pression atmosphérique est, selon lui, la force de l'air qui, comprimé par l'attraction mutuelle entre la Terre et les matières terrestres, veut au contraire se raréfier, ce qui rend difficile l'explication de la raison pour laquelle, au sommet de la montagne, le mercure ne monte pas autant qu'à son pied. Sceptique quant au bon résultat de l'expérience du Puy de Dôme avant sa réalisation, mais mis face au grand succès de l'expérience, Roberval a inventé, au terme de la réflexion, une hypothèse se fondant sur la force de l'attraction mutuelle.

La pression des éléments est plus grande à la surface de la terre qu'à distance de cette surface lorsqu'on s'élève en l'air; et la même chose se produira dans le système le plus vaste, dont toutes les parties seront portées les uns vers les autres par une action mutuelle et réciproque, de manière à demeurer étroitement liées; en particulier si au centre d'un tel système (ce qui a lieu dans le nôtre) on place celui des corps composants un tel système qui exerce l'action la plus forte. ${ }^{11}$

Roberval ainsi attribue la différence de la pression atmosphérique à celle de l'attraction mutuelle qui est en raison inverse de la distance du centre de la Terre.

C'est Jean Pecquet qui est le premier à se rendre compte du fait qu'il faut mettre en considération les deux facteurs, l'élasticité de l'air et la pesanteur de l'air, pour bien expliquer le mécanisme de la pression atmosphérique. Le livre de Pecquet, paru en 1651, trois ans avant la rédaction des Traités de l'équilibre des liqueurs et de la pesanteur de la masse de l'air, permet de bien aborder l'argumentation des textes de Pascal.

\subsection{La modélisation par Jean Pecquet}

Les Experimenta nova anatomica de Jean Pecquet, physiologue français, connu parmi les pascaliens comme celui qui présente l'expérience du vide dans le vide effectuée par Auzout, n'a pas en effet pour objectif de rapporter les expériences curieuses d'Auzout, mais de démontrer de façon convaincante que la pression atmosphérique se produit par l'effet de la pesanteur de l'air et de l'élasticité de l'air ${ }^{12}$.Avant de montrer les expériences qui en témoignent, cette œuvre, en disant que «Esse non pondus tantum, sed \& rarefactorium Aeri Elaterem Experimentis demonstratur ", dessine un modèle de la Terre, entourée d'abord d'une couche d'eau, ensuite de la masse de l'air, qui, toutes les deux, veulent descendre au centre de la Terre. La masse de l'air, qualifiée de « laine ou éponge ", est comprimée dans la partie inférieure par son propre poids, 
Hunc fingito tibi velut spongiosi vel lanei potius cumuli terraqueum Orbem ambientis molem; cujus proinde partes superiores ab inferioribus, compressione gradatim incedente, sustineantur, sicut, quo Terris accedunt vicinius, eo etiam incumbentium nisu \& pondere compactius opprimantur: \& ob id spontaneo dilatatu (quem Elaterem nuncupo) utcunque graviter aggestarum onus, subjectae nitantur, si libertas adsit, rarescere.

Hinc infero ejuscemodi partium infimam, ut toti subjectam oneri, sic omnium esse maxime condensatam ; \& per hoc ab eadem non solo duntaxat Pondere, sed \& Elasteris, cujus tum validissimus ad rarefaciendum nixus est, virtute Terraqueae Sphaerae premi superficiem. ${ }^{13}$

Après avoir montré un modèle de la Terre entourée de "la laine » (ce qui est très proche du modèle de Pascal), Pecquet, en invoquant trois expériences, démontre le mécanisme de la pression atmosphérique. Pecquet, tout d'abord, commence par déclarer que l'air a une qualité immanente d' élasticité », en rappelant l'expérience de la vessie de carpe ${ }^{14}$; et ensuite, se fondant sur l'expérience du Puy de Dôme, une variation de l'expérience de la vessie de porc, il affirme que la pression atmosphérique de l'air est variable selon l'altitude au-dessus du niveau de la mer; d'où l'auteur tire la conclusion que l'altitude au-dessus de la mer, c'est-à-dire la pesanteur de l'air accumulé détermine évidemment la valeur de la pression atmosphérique ${ }^{15}$. A la fin, afin de renforcer cette idée, l'auteur fait appel à l'expérience du vide dans le vide effectuée par Auzout en tant qu'état idéal où l'air est infiniment raréfié ${ }^{16}$.

\section{L'attitude de Pascal par rapport à l'élasticité de l'air}

\subsection{Pascal apprivoise l'élasticité de l'air}

Pascal, tout en qualifiant la masse d'air de "grand amas de laine ", ainsi que Pecquet le fait, montre, au lieu de l'expérience de la vessie de carpe, celle d'un ballon à demi rempli d'air qu'on porte sur le sommet d'une montagne, expérience fort probablement motivée par la vessie de carpe de Roberval. Il nous faut donc comparer, avec l'expérience de la vessie de carpe, l'expérience du ballon désenflé que Pascal a porté au sommet du Puy de Dôme, afin de vérifier, dans le monde terrestre, l'expérience de Roberval réalisée dans un état idéal. La ressemblance entre le développement de l'argumentation chez Pascal et chez Pecquet ne signifie pas pour autant que Pascal admet l'élasticité de l'air, que ce soit dans la vessie de carpe, ou dans le ballon à demi rempli d'air. L'explication de Pascal est la suivante.

Comme il arriverait en un grand amas de laine, si on en avait assemblé de la hauteur de vingt ou trente toises, que cette masse se comprimerait elle-même par son propre poids, et que celle qui serait au milieu, ou près du haut, parce qu'elle serait pressée d'une plus grande quantité de laine, ainsi la masse de l'air, qui est un corps compressible et pesant aussi bien que l'air, se comprime elle-même par son propre poids; et l'air qui est au bas, c'est-à-dire dans les lieux profonds, est bien plus comprimé que celui qui est plus haut, comme aux sommets des montagnes, parce qu'il est chargé d'une plus grande quantité d'air.

Comme il arriverait en cette masse de laine que, si on prenait une poignée de celle qui est dans le fond, dans l'état pressé où on la trouve, et qu'on la portât, en la tenant toujours pressée de la même sorte, au milieu de cette masse, elle s'élargirait d'elle-même, étant plus proche du haut, parce qu'elle aurait une moindre quantité de laine à supporter en ce lieu-là, ainsi, si l'on portait de l'air, tel qu'il est ici-bas, et comprimé comme il y est, sur le sommet d'une montagne, par quelque artifice que ce soit, il devrait s'élargir lui-même, et devenir au même état que celui qui environnerait sur cette montagne, parce qu'il serait chargé de moins de l'air en cet 
endroit-là qu'il n'était au bas; et par conséquent, si on prenait un ballon à demi plein d'air seulement, et non pas tout enflé, comme ils le sont d'ordinaire, et qu'on le portât sur une montagne, il devrait arriver qu'il serait plus enflé au haut de la montagne, et qu'il devrait s'élargir à proportion de ce qu'il serait moins chargé (.... ${ }^{17}$

Le rapport rédigé par Pascal met l'accent non pas sur l'élasticité de l'air dans le ballon, mais sur le fait que la pression de l'atmosphère faiblit, au fur et à mesure que l'altitude augmente et, par conséquent, que l'air dans le ballon, étant comprimé moins fortement, s'élargit proportionnellement à la diminution de la pression atmosphérique. Pascal, à la différence de Pecquet, s'abstient, totalement de faire intervenir la propriété d'élasticité de l'air, et ne parle que de la nature de "compression » de l'air et de la tendance du ballon à "s'élargir » lorsque la force de compression diminue. Ce mode d'explication permet de sauver le phénomène sans recourir à l'hypothèse de l'élasticité, hypothèse qui provoquerait un débat concernant la théorie des matières, sujet métaphysique et traditionnel dans lequel Pascal ne souhaite pas s'engager ${ }^{18}$.

Ce qui est caractéristique dans ce processus, ce n'est pas que Pascal fasse intervenir la causalité physique dans l'explication de la propriété physique (par exemple « la masse d'inertie» en tant que cause du "mouvement d'inertie»), mais qu'il tire profit du modèle de la balance par lequel deux quantités sont comparées au niveau du phénomène. Dans le problème dont il s'agit, Pascal montre aux lecteurs l'élasticité de l'air, non comme nature immanente au corps, ni comme cause supposée de la pression atmosphérique, mais comme des formes d'états équilibrés entre la pression atmosphérique et la pression intérieure du ballon; en d'autres termes, il veut, à travers un ballon enflé ou désenflé s'équilibrant avec la pression atmosphérique, que «tous portent la pensée vers le même objet ${ }^{19}$ ", non pas vers « la nature » des choses.

Pascal en outre, évitant soigneusement les verbes comme "se dilater" ou "se raréfier ", n'utilise, ici, que le verbe "s'élargir» pour s'en tenir à des mots qui n'évoquent pas la notion de l'élasticité. Ainsi essaie-t-il de limiter au minimum le nombre des hypothèses, savoir l'hypothèse de la pesanteur de l'air (qui n'était pas unanimement acceptée) et celle de la pression causée par la pesanteur de l'air.

Il convient toutefois de s'abstenir d'accorder trop d'importance à Pascal. Il nous faut nous demander si un moindre nombre de principes répond de façon adéquate aux questions posées par les expériences de Roberval. Le cadre de pensée de Pascal, certes, pouvait donner une explication irréprochable de l'expérience de la vessie de carpe (ou de l'expérience du ballon au Puy de Dôme). Mais ce cadre de pensée ne pouvait pas expliquer, par exemple, l'expérience dans laquelle les mêmes quantités d'eau et d'air sont placées dans des tubes en apparence vides, parce que, la pesanteur étant la seule cause de la pression des liqueurs selon Pascal, l'air n'exerce jamais une pression plus forte que l'eau. Les deux principes de Pascal, la pesanteur et la pression ne sont pas en effet en mesure de répondre pleinement à ce grand problème de l'époque. Bien que Pascal «montre » au niveau du phénomène l'élasticité de l'air, elle ne cadre pas bien dans sa théorisation. Les Traités de l'équilibre des liqueurs et de la pesanteur de la masse de l'air, tout en ayant, à nos yeux, une teneur complètement achevée et un développement logique magnifiquement élaboré, n'ont pas été publiés de son vivant. Ne peut-on pas imaginer que cela est dû certes à la " conversion définitive » de son auteur, mais aussi à son insuffisance théorique? 


\subsection{Le dispositif de l'expérience du vide dans le vide chez Roberval et Pascal} pourtant toutes les problématiques concernant la raréfaction et la condensation dont Roberval s'était occupé. Au moment où nous avons ôté le doigt qui bouchait l'orifice supérieur du tuyau, l'air est entré tout d'un coup dans le tuyau et le vif-argent est remonté rapidement. Tout en bannissant le problème de la densité de l'air, Pascal s'est concentré sur l'équilibre des liqueurs principalement dans son dernier ouvrage. Le deuxième dispositif de Pascal, considéré comme "manifestation scientifique » qui, omettant les phénomènes périphériques, présente seulement l'essentiel «pour Pascal », nous semble excellent même s'il s'agit seulement d'une expérience fictive.

Pascal, à vrai dire, aurait eu l'idée de l'élasticité de l'air dans les premiers moments de ses recherches sur l'hydrostatique, puisque, dans le premier dispositif de Pascal, «le petit trou » percé dans la vessie de porc qui couvre l'orifice supérieur du tuyau permet le changement graduel de la densité de l'air dans le tuyau. Mais avec son deuxième dispositif, Pascal coupe toute voie vers l'étude de la densité et de la raréfaction de l'air. Et il nous apparaît que les Traités de l'équilibre des liqueurs et de la pesanteur de la masse de l'air, évitant expressément ces questions pour ne pas se plonger dans une discussion les concernant, développe son argumentation en faisant un choix de terminologie. 


\section{Conclusion}

51 L'élasticité de l'air, depuis la correspondance entre Torricelli et Ricci, était un grand problème dans l'étude de la pression atmosphérique. Cette notion très importante dans l'histoire de l'hydrostatique a été reprise en France quatre ans plus tard surtout par Roberval. Toutefois, l'argumentation de Roberval, très expérimentale sur un certain plan, mais spéculative sur un autre, n'est pas parvenue à éclaircir totalement la question de la pression atmosphérique. Pascal, évitant habilement une notion physique aussi dangereuse, s'en est tenu à soutenir « la pesanteur de l'air » comme principe de l'hydrostatique, dans le cadre d'un mécanisme assez rigoureux. Le développement de l'argumentation chez Pascal, d'une part très solide et ferme grâce à l'expulsion de toute qualité occulte, n'est pas parvenu, d'autre part, à saisir dans toute son ampleur la nature des liqueurs élastiques.

\section{BIBLIOGRAPHIE}

UCHIDA, Masao, « De la faisabilité des Expériences du vide dans le vide rapportées par Pascal et ses contemporains ", Bulletin de la Faculté des Lettres et Sciences Humaines de Université Wakô, no. 17, 1982, (en japonais). La traduction anglaise par l'auteur « The feasibility of "les Expériences du vide dans le vide" as contrived by Pascal and his contemporaries » in Réflexions sur la place de Descartes et de Pascal dans l'histoire de la pensée scientifique et Etude des bibliographies concernées, p. 107-143 (Actes des études coopératives en 2004-2006, organisées par Kimiyo KOYANAGI, professeur à l'Université Préfectorale d'Aichi).

DESCOTES Dominique, « Le vide dans le vide », in XVII ${ }^{e}$ siècle, n. 207, 52 année, n. 2-2000, pp. 257-272.

KOYANAGI, Kimiyo, Pascal, de l'intuition à l'affirmation, Presse universitaire de Nagoya, 1992, (en japonais).

KOYANAGI, Kimiyo, «Pascal et l'expérience du vide dans le vide ", Japanese Studies in the History of Sciences 17, 1978.

KOYANAGI, Kimiyo, « Les expériences du vide dans le vide », in Courrier du CIBP nº 11-1989, p. 2-23)

ROMEO Maria Vita, Il Numero e l'Infinito, L'itinerario pascaliano dalla scienza alla filosofia, C.U.E.C.M., Catania, Italia. Voir surtout p. $132-140$.

\section{NOTES}

1. «Maintenant, si nous enfoncions de force un plan coupant, FG, qui puisse entrer et couper la laine comprimée, je dis que si la laine FBCG était comprimée comme précédemment, quoique le fond $\mathrm{BC}$ ne sente plus rien du poids imposé par le plomb $\mathrm{E}$, de toute façon il supportera le même poids qu'il supportait auparavant.» (Trad. française de A. Beaulieu). Correspondance du P. 
Mersenne, publiée et annotée par Cornelis de Waard et Armand Beaulieu, XIII, Fin juillet 1644, p. 182.

Figure extraite de la Correspondance du P. Mersenne

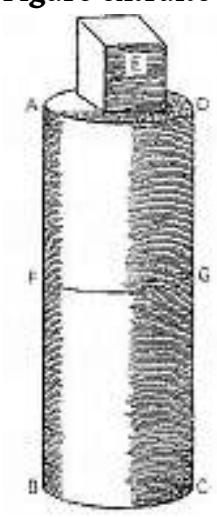

2. En ce qui concerne la diffusion de ces extraits des lettres de Torricelli et leur influence sur la France, voir KOYANAGI Kimiyo, «De l'intuition a l'affirmation: Cheminement de Pascal jusqu'à l'achèvement de ses CEuvres de physique ", Etudes de Langue et Litterature Francaises, $\mathrm{N}^{\circ} .60$, Société Japonaise de Langue et Littérature Françaises, Tokyo, 1992, pp. 44-56. « Cet effrayant petit livret... Expériences nouvelles touchant le vide de Blaise Pascal ", in Les Pascal à Rouen 1640-1648, Colloque de l'Université de Rouen, 17, 18, 19 novembre 1999, Textes réunis par Jean-Pierre Clero, Publications de l'Université de Rouen, 2001, pp. 137-157.

3. Voir notre DVD. Bien que nous ayons versé aussi attentivement que possible le mercure, en nous servant d'un entonnoir, nous n'avons pas pu empêcher l'air d'entrer dans le tuyau rempli de mercure.

4. Gilles Personne de Roberval, De Vacuo Narratio ad Nobilem Virum Dominum Desnoyer in Cuvres de Blaise Pascal, publiées par L. Brunschvicg, P. Boutroux et F. Gazier, 14 vol. , 1904-1914, Collection des Grands Écrivains de la France (abrégées dans notre article en GEF), II, p. 329. Blaise Pascal Cuvres complètes, établies, présentées et annotées par Jean Mesnard, Desclée de Brouwer «Bibliothèque européenne », 1964, p. 609 (abrégées en OC).

5. Voir les commentaires par V. Jullien dans Elément de géométrie de G. P. de Roberval, textes présentés par Vincent Jullien, Vrin, 1996, pp. 460-461.

6. Voir GEF, p. 313-318. Le développement de la recherche de Roberval relative à ces expériences, à partir de la Première Narration jusqu'à la Seconde Narration est analysé de façon chronologique dans Léon Auger, Un savant méconnu: Gilles Personne de Roberval (1602-1675), A. Blanchard, 1962, pp.117-133, suivant lequel Roberval a effectué les deux expériences comparatives avant l'expérience de vessie de carpe.

7. Bien que R. Boyle, par exemple, ait eu connaissance de l'expérience de la vessie de carpe en 1660 (The works of Robert Boyle, edited by Michael Hunter and Edward B. Davis, London, Pickering \& Chatto, 1999-2000, 14 vol, t. 1, p. 174), il ne s'était pas encore procuré le De vacuo narratio de Roberval en 1662 (t. 3, p. 11). Je remercie infiniment M. Yoshimoto Hideyuki, professeur à l'Université des Langues Étrangères, de m'avoir donné ces renseignements très précieux.

8. Gille Personne de Roberval, De mundi Systemate, partibus, \& motibus eiusdem, 1644, p. 3. Il avait déjà proposé, dans la lettre écrite en 1635 avec É. Pascal ( Lettre de Messieurs Pascal et de Roberval à M. de Fermat, 16 août 1636, OC, éd. J. Mesnard, II., p. 129) l'idée de l'attraction mutuelle, quoi que ce soit une hypothèse qui explique la cause de la pesanteur, et la maintint jusqu'en 1669 lors du débat sur la cause de la pesanteur à l'Académie Royale des Sciences, in Euvres complètes de Christiaan Huygens, publiées par la société Hollandaise des sciences, t. XVI, Swets \& Zeitlinger. N. V., Amsterdam, 1888-1950, t. XIX, p. 628.

9. Jean-Pierre Fanton d'Andon, L'Horreur du vide, CNRS, 1978, p. 4-15 
10. Sur ce point, voir Vincent Jullien, Philosophie naturelle et géométrie au XVII ${ }^{e}$ siècle, Honoré Champion, 2006, p. 205-208.

11. GEF. II, p. 360-361. OC II, p. 611.

12. Sur le rôle joué de Pecquet dans l'histoire des sciences surtout en Angleterre, voir Charles Webster, "The Discovery of Boyle's Law and the Concept of the Elasticity of Air in the Seventeenth Century ", Archive for the History of Exact Sciences, 21 (1965), 441-502.

13. Jean Pecquet, Experimenta Nova Anatomica, Paris, 1651, p. 89. «Imaginez cela (sc. l'air) comme un amas d'éponge ou plutôt de laine entourant le globe terrestre, dont par conséquent les parties supérieures sont soutenues par les inférieures, avec une compression croissant par degrés, de telle manière que, plus elles se trouvent proches de la terre, plus aussi elles sont comprimées de façon compacte par la pression et le poids de celles qui pèsent sur elles; et à cause de cette charge de choses lourdement amassées, elles s'efforcent par une dilatation spontanée (que j'appelle elater), de se raréfier, si elles en ont la liberté. De là j'infère que la plus basse des parties, étant soumise à tout le poids, est ainsi la plus condensée de toutes, et que c'est par la même force que la surface de la sphère terraquée est pressée, savoir non pas seulement par le poids, mais aussi par l'elater, dont l'effort très puissant tend à la raréfaction. » Je remercie profondément $\mathrm{M}$. Honma Eio de m'avoir offert la microfiche de ce texte.

14. Experim. I : «Vacuata vesicula sponte ad Hydrargyri lapsum, intra sublimem tubi ampullam rarescens, Rarefactorium Aeris indicat Elaterem », Ibid, p. 91.

15. Experim. II «Compactiores esse gradatim subjectas incumbentibus Aeris partes, diversus Hydrargyri lapsus, pro diversa montis altitudine probat. », Ibid, p. 99.

16. Experim. III « Exterioris Aeris cum inferiori Hydrargyri cylindra aequipondium ostenditur. », Ibid., p. 102.

17. MES. II, p. 1063-1064 (souligné par nous).

18. Jean Mesnard, «La pesanteur de l'air chez Pascal et Roberval », in Die Schwere der Luft in der Diskussion des 17. Jahrhunderts, Hrsg. von Wim Klever. Published : Wiesbaden : Harrassowitz, 1997, p. 47-72.

19. Pascal, De l'esprit géométrique, OC, III, p. 397.

20. OC II, p. 607.

\section{INDEX}

Mots-clés : Pascal, vide

Keywords : Pascal, vacuum

Index géographique : Japon

\section{AUTEUR}

\section{HIROKI TAKEDA}

Université Saint Thomas d'Osaka, Japon 